Preprint submitted to $V U V-13$

\title{
BULK BAND GAPS IN DIVALENT HEXABORIDES: A SOFT X-RAY EMISSION STUDY
}

\author{
J. D. DENLINGER \\ Advanced Light Source, Lawrence Berkeley National Lab \\ Berkeley, CA 94720, USA \\ E-mail: JDDenlinger@lbl.gov \\ G.-H. GWEON, J. W. ALLEN \\ Randall Laboratory of Physics, University of Michigan \\ Ann Arbor, MI 48109-1120, USA \\ A. D. BIANCH市, Z. FISK \\ National High Magnetic Field Lab, Florida State University \\ Tallahassee, FL 32306, USA
}

\begin{abstract}
Boron K-edge soft x-ray emission and absorption are used to address the fundamental question of whether divalent hexaborides are intrinsic semimetals or defect-doped bandgap insulators. These bulk sensitive measurements, complementary and consistent with surface-sensitive angle-resolved photoemission experiments, confirm the existence of a bulk band gap and the location of the chemical potential at the bottom of the conduction band.
\end{abstract}

\section{Introduction}

The discovery of weak itinerant ferromagnetism in certain divalent hexaborides 1 , vides strong motivation to determine the underlying electronic structure giving rise to the metallic carriers. One possibility predicted by LDA band calculations 5 , 6 , and supported by the interpretation given to magneto-oscillatory studies is a semi-metallic band overlap at the $\mathrm{X}$-point of the cubic Brillouin zone, the absence of which would render stoichiometric material to be insulating. Several theoretical discussions, 0,11 presume the existence of such an overlap. As summarized elsewhere 12 we have recently given conclusive experimental proof that there is instead an X-point gap. This result eliminates all models that assume overlap and so may favor a ferromagnetic dilute electron gas picture 13 , but it also forces the consideration of boron vacanies as the origin of the electrons observed in nominally stoichiometric divalent materials, possibly favoring a picture in which the magnetic moments are car- ried by boron vacancies 14 .

The X-point gap was first observed in angle resolved photoemission (ARPES) of $\mathrm{EuB}_{6}$ and $\mathrm{SrB}_{6}$ and ascribed by us initially only to the surface region probed in ARPES 15 . Strong motivation to reinterpret the ARPES result as showing a bulk gap 16 was provided by a recent band calculation 17 that includes a GW self energy correction and predicts $\mathrm{CaB}_{6}$ to have the X-point band gap of $0.8 \mathrm{eV}$ similar to that measured by ARPES. Results from bulk sensitive soft $\mathrm{x}$-ray emission and absorption spectroscopy (SXE and XAS, respectively) showing the gap are an essential part of our experimental proof. This paper gives important aspects of the SXE/XAS results and analysis that were not presented previously.

\section{Experimental}

Single crystal hexaboride samples were grown from an aluminum flux using powders prepared by boro-thermally reducing cation oxides, a method shown to yield high quality with regard to 
both structure and chemical composition 18 . Soft x-ray emission and absorption experiments were performed at the ALS Beamline 8.0.1 using the Tennessee/Tulane grating spectrometer. The experimental emission and absorption spectral resolutions were $\approx 0.35 \mathrm{eV}$ and $\approx 0.1 \mathrm{eV}$, respectively. SXE, measured with a 1500 line/mm grating for fixed photon energy excitation at and above the B K-edge threshold, is used as a probe of the occupied boron partial density of states for dipoleallowed transitions back to the $\mathrm{B} 1 s$ core-level, i.e. $p$-states. X-ray absorption, a probe of the unoccupied states, was measured both with total electron yield (TEY) as a function of photon energy and also with partial fluorescence yield (PFY) with the detection window covering the entire valence band emission. Differences between TEY and PFY signals arise from differing attenuation lengths and the experimental geometry which was set to $60^{\circ}$ incidence excitation and $30^{\circ}$ grazing emission relative to the sample surface. Absolute PFY energies were calibrated to published TEY spectra of the hexaboride 10 and SXE spectra were calibrated to the excitation energy via the presence of elastic scattering in the emission spectra. Previous very early work on the hexaborides using electron-excited soft x-ray emission and thin film absorption measurements 20121 do not provide clear enough spectral detail at threshold to address the band gap issues that have only recently been raised.

\section{Results}

Fig. 1 shows a representative data set of soft $\mathrm{x}$-ray emission and absorption at the boron $\mathrm{K}$ edge for the divalent hexaborides. Very similar data to this example from a cleaved $\mathrm{YbB}_{6}$ sample was also obtained for $\mathrm{CaB}_{6}, \mathrm{SrB}_{6}$, and $\mathrm{EuB}_{6}$. Fig. 1(a) compares the two methods of measuring x-ray absorption, TEY and PFY. TEY, a measurement of the sample current, exhibits a sharp peak at $\approx 194 \mathrm{eV}$ corresponding to a well-known B $1 s \rightarrow 2 p \pi^{*}$ transition 19 , which in part arises from surface layer oxidation of the air-cleaved sample. Also the absolute TEY signal exhibits a high background (removed in Fig. 1(a)) with declining slope due to the presence of lower energy absorption edges.
In contrast, the PFY signal, a measure of valence emission intensities integrated over the energy window shown in Fig. 1(b), does not exhibit the sharp TEY absorption peak due to a greater bulk emission sensitivity than the excitation depth which rapidly decreases while scanning through the absorption resonance. Also the valence band PFY signal inherently has zero prethreshold intensity and hence is preferred over TEY for careful measurement of the threshold region. The PFY spectrum for $\mathrm{YbB}_{6}$ shows a weak step-like threshold onset at $187.1 \mathrm{eV}$ which is highlighted by a logarithmic scale in the inset to Fig. 1(a). TEY also shows an intensity cusp at this energy, but only for cleaved surfaces with minimal surface contamination. We interpret this threshold onset in the PFY spectrum as the energy position of the chemical potential which we define at the half-step intensity. This location of the chemical potential or Fermi-edge $\left(E_{\mathrm{F}}\right)$ in the conduction band is immediately important for electronic structure models and is consistent with negative Hall coefficient measurements indicating the presence of electron (and not hole) carriers in the bulk22 23.

Valence band emission spectra, shown in Fig. 1(b) were acquired at selected photon energies indicated by arrows in Fig. 1. An elastic peak present in the emission spectra is resonantly enhanced at the $\mathrm{B} 1 s \rightarrow 2 p$ absorption peak and is used for calibration of the SXE energy scale to that of TEY and PFY spectra. Excitation at the selected peaks in the PFY/TEY spectra and far above threshold show similar valence band emission profiles with small variations in the relative intensities of at least six discernable peaks and shoulders. Threshold excitation on the other hand produces much larger variation in the relative intensities (and energies) of the different valence emission peaks (discussed in the next section). The elastic peak is also observed to be enhanced at threshold, thus providing a distinct marker of $E_{\mathrm{F}}$ for all the emissionspectra. The non-threshold SXE spectra, on the other hand, exhibit a strong non-metallic decay of intensity approaching $E_{\mathrm{F}}$, in contrast to the weak steponset in the PFY spectrum which implies a small occupancy of states at $E_{\mathrm{F}}$. A lack of clear SXE detection of such density of states near $E_{\mathrm{F}}$ could 
be due to too little occupancy, very weak boron $p$-character, and/or a poorer SXE resolution than the occupied bandwidth.

\section{Comparison to band theory}

A detailed comparison of the $\mathrm{YbB}_{6} \mathrm{SXE}$ and PFY spectra to both LDA and GW calculations, presented in Fig. 2, reveals a clear distinction between the band-overlap and band gap electronic structure models. Available partial density of states (DOS) calculations exist in the literature for $\mathrm{SrB}_{6}$ only 6 and the GW calculation was performed for $\mathrm{CaB}_{6}$ 17. However, since experimental SXE bandwidths of all hexaborides are very similar and the theoretical bandwidths for a given calculation method are also similar between different hexaborides, the comparison of experiment to theory involving different divalent cations is justified.

Fig. 2(a) shows the combined SXE (194.1 eV excitation) and PFY spectra with the chemical potential shifted to $0 \mathrm{eV}$ and with comparison to the theoretical LDA $p$-DOS for $\mathrm{SrB}_{6}$. The relative SXE and PFY intensities have been scaled to match the theoretical DOS amplitudes above and below $E_{\mathrm{F}}$. Boron $s$-DOS and cation $d$-DOS, not probed by these measurements, are strongest at $-8 \mathrm{eV}$ and above $+5 \mathrm{eV}$, respectively. The corresponding $k$-resolved LDA band structure (for $\mathrm{CaB}_{6}$ ) along $\Gamma-\mathrm{X}$ for these DOS, exhibiting a small band overlap at the $\mathrm{X}$-point between the valence and conduction bands, is plotted in Fig. 2(b). A clear discrepancy between the energy position of the LDA occupied and unoccupied DOS with the experimental spectra is seen.

A rigid shift of the theoretical DOS to lower energy provides a better agreement with experiment. The overall occupied boron-block bandwidth and relative peak amplitudes are in good agreement with the LDA $p$-DOS. However, independent energy shifts of the occupied and unoccupied states $(-0.8 \mathrm{eV}$ and $-0.5 \mathrm{eV}$, respectively) is required to make best alignment to the 2.5-5 eV SXE and $3 \mathrm{eV}$ PFY peaks (Fig. 2(c)). This suggests a relatively larger experimental energy separation between the valence and conduction bands than predicted by LDA and thus no Xpoint band overlap.
Fig. 2(d) shows the GW bands along $\Gamma-\mathrm{X}$ for $\mathrm{CaB}_{6}$ exhibiting a band gap at the $\mathrm{X}$-point. The GW band calculation predicted a $10 \%$ expansion of the boron-block bandwidth compared to LDA. However this larger GW bandwidth is not consistent with the bandwidths measured here by SXE or by ARPES 12 which are in better agreement with LDA. Hence for Fig. 2(d) the GW energy scale has been multiplied by 0.9 to match the LDA bandwidth, and then shifted to lower energy placing $E_{\mathrm{F}}$ at the bottom of the X-point conduction band consistent with the PFY measurement.

Since the divalent hexaboride conduction band minimum resides at the X-point, threshold excitation into these unoccupied states is expected to exhibit X-point $k$-selectivity in the emission process due to the lack of intermediate scattering paths to other lower energy $k$ points. Indeed, a favorable one-to-one correspondence can be made between the X-point band energies of the modified GW band calculation in Fig. 2(d) to the dominant peaks labeled $(a-e)$ in the threshold-excited SXE spectrum plotted in Fig. 2(e). Most important for our goal of distinguishing band-overlap versus band-gap electronic structure is the identification of peak $a$ as the boron-block valence band maximum at $\approx 1$ eV below $E_{\mathrm{F}}$. The threshold-excited $\mathrm{YbB}_{6} \mathrm{SXE}$ spectrum thus provides a direct quantification of the bulk bandgap to be $1.0 \mathrm{eV}$ minus the energy that the conduction band dips below $E_{\mathrm{F}}$, thus providing a firm basis for the reasoning set forth in 12 .

\section{Discussion}

These soft x-ray measurements verifying the existence of a bulk band gap are complementary to our surface-sensitive ARPES measurements of the $\Gamma-\mathrm{X}$ band structure 12,15 which provide a more detailed view of the $\mathrm{X}$-point gap. The position of the chemical potential at the bottom of the conduction band is also qualitatively consistent with ARPES measurements of an electron pocket at the X-point. While excess electron concentrations at the surface can be explained by band bending and charge redistribution effects, excess electron carriers in the bulk forces one to confront 


\section{J. D. Denlinger et al.}

the issue of off-stoichiometry defects, i.e. electron counting and band filling of the hexaboride band structure with the presence of a band gap predicts divalent materials to be insulators. A rigid covalently bonded boron sublattice with mobile cations is suggestive of cation vacancies only, i.e. hole carriers, inconsistent with Hall coefficient measurements. The presence of boron vacancies on the other hand appears to be a more natural source of excess electrons and such defects have even been recently shown theoretically to possess magnetic moments which may be highly relevant to the origin of the anomalous ferromagnetism in La-doped $\mathrm{CaB}_{6}$ and other divalent hexaboride systems. 14 .

We have also found from a combination of SXE/PFY and ARPES spectra12,24 that the Xpoint gap is absent for trivalent $\mathrm{LaB}_{6}$ and mixed valent $\mathrm{SmB}_{6}$ implying a non-trivial transformation from divalency to trivalency. Additionally a distinct anomalous deviation from Vegard's law exists in the $\mathrm{Ca}_{1-x} \mathrm{La}_{x} \mathrm{~B}_{6}$ series. Rather than a monotonic increase in lattice constant expected from the larger $\mathrm{La}^{3+}$ size, the lattice parameter at first shrinks to a minimum at $\approx 10 \%$ La doping before increasing again at higher La-doping 25. This critical La concentration may be correlated with a crossover from band-gap to band-overlap $\mathrm{X}$-point electronic structure.

\section{Summary}

Boron K-edge soft x-ray emission and absorption have been used to probe boron occupied and unoccupied partial density of states of the divalent hexaborides, with data from $\mathrm{YbB}_{6}$ used as an example. The chemical potential is identified to be located at the bottom of the conduction states in the absorption spectra and comparison of the emission spectra to calculated LDA density of states is used to identify the energy of the valence band maximum to be $\approx 1 \mathrm{eV}$ below the chemical potential. This result establishes the existence of a bulk X-point band gap consistent with recent GW band calculations and rules out the band overlap model as a starting point for theories to explain the novel ferromagnetism in doped and undoped hexaborides.

\section{Acknowledgments}

This work was supported at $\mathrm{U}$. of Michigan by the U.S. DoE under Contract No. DEFG02-90ER45416 and by the U.S. NSF Grant No. DMR-99-71611. The ALS is supported by the U.S. DoE under contract No. DE-AC03$76 \mathrm{SF} 00098$.

\section{References}

[†] present address: Los Alamos National Laboratory, Los Alamos, NM 87545

1. D. P. Young et al., Nature 397, 412 (1999).

2. P. Vonlanthen et al., Phys. Rev. B 62, 10076 (2000).

3. H. R. Ott et al., Physica B 281-2, 423 (2000).

4. T. Terashima et al., J. Phys. Soc. Jpn. 69, 2423 (2000).

5. A. Hasegawa and A. Yanase, J. Phys. C, Solid State Phys. 12, 5431 (1979).

6. S. Massidda, A. Continenza, T. M. D. Pascale, and R. Monnier, Z. Phys. B 102, 83 (1997).

7. R. G. Goodrich et al., Phys. Rev. B 58, 14896 (1998).

8. M. C. Aronson et al., Phys. Rev. B 59, 4720 (1999).

9. M. E. Zhitomirsky, T. M. Rice, and V. I. Anisimov, Nature 402, 251 (1999).

10. L. Balents and C. M. Varma, Phys. Rev. Lett. 84, 1264 (2000).

11. V. Barzykin and L. P. Gor'kov, Phys. Rev. Lett. 84, 2207 (2000).

12. J. D. Denlinger et al., cond-mat/0107429.

13. D. Ceperley, Nature 397, 386 (1999).

14. R. Monnier and B. Delley, cond-mat/010521d.

15. J. D. Denlinger et al., cond-mat/000922.

16. J. D. Denlinger et al., Bull. Am. Phys. Soc. 46, 1218 (2001).

17. H. J. Tromp et al., Phys. Rev. Lett. 87, 016401 (2001).

18. H. R. Ott et al., Z. Phys. B 102, 337 (1997).

19. J. J. Jia et al., J. Electron Spectrosc. Related Phenom. 80, 509 (1996).

20. I. I. Lyakhovskaya, T. M. Zimkina, and V. A. Fomichev, Sov. Phys. Solid State 12, 138 (1970).

21. M. Okusawa, K. Ichikawa, T. Matsumoto, and K. Tsutsumi, J. Phys. Soc. Jpn. 51, 1921 (1982).

22. Z. Fisk J. Appl. Phys. 50, 1911 (1979).

23. J. M. Tarascon J. Appl. Phys. 51, 574 (1980).

24. S.-K. Mo et al., cond-mat/0107203.

25. A. D. Bianchi and Z. Fisk, unpublished. 


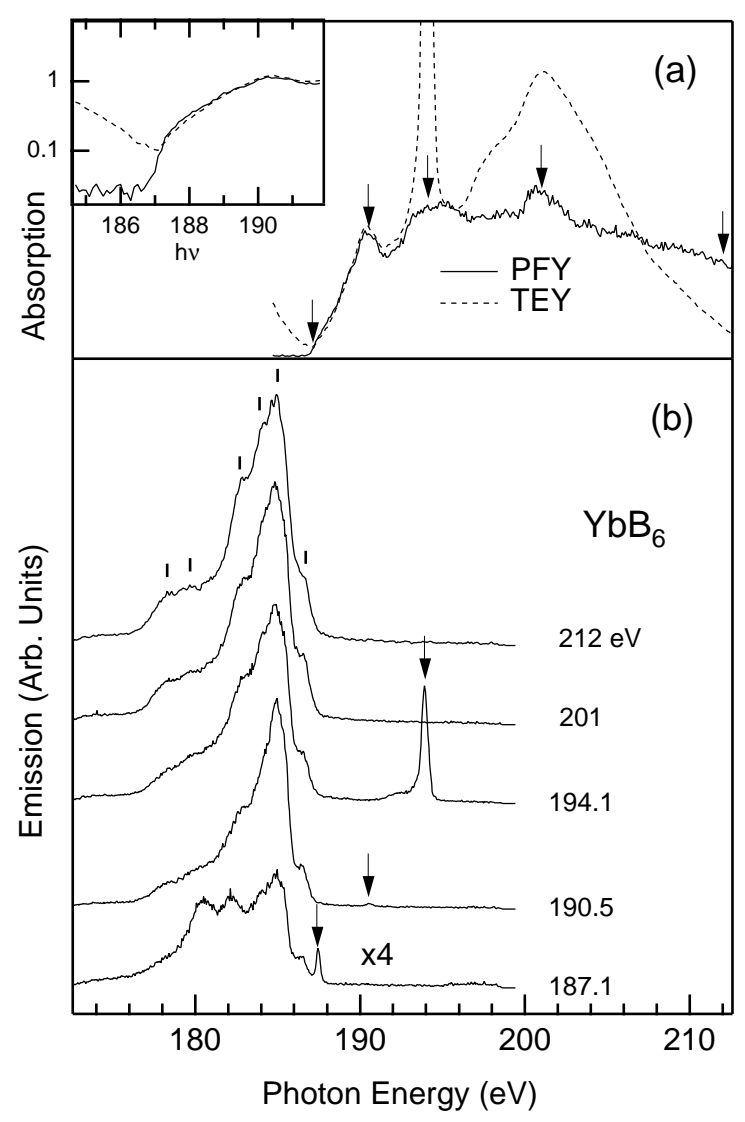

Fig. 1. Soft x-ray absorption (TEY, PFY) and emission (SXE) boron K-edge data set for $\mathrm{YbB}_{6}$. Arrows and values indicate the excitation energies. The logarithmic intensity scale of the inset highlights the step intensity onset of the PFY signal.

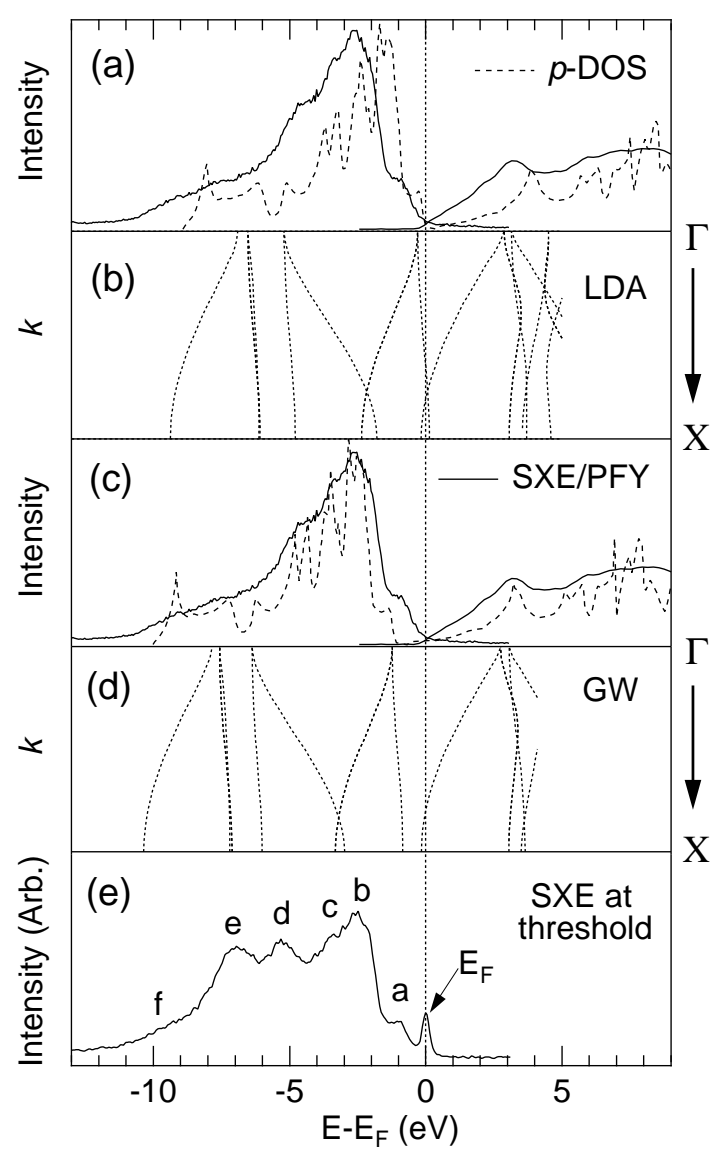

Fig. 2. (a) Comparison of $\mathrm{YbB}_{6} \mathrm{SXE}$ and $\mathrm{PFY}$ to LDA boron $p$-DOS for $\mathrm{SrB}_{6}$, (b) LDA band structure along $\Gamma$-X exhibiting band overlap at X, (c) comparison of SXE and PFY to energy shifted boron $p$-DOS, (d) GW band structure along $\Gamma-\mathrm{X}$ exhibiting a band gap, (e) $\mathrm{YbB}_{6}$ SXE excited at threshold exhibiting $\mathrm{X}$-point $k$-selectivity of peaks $(a-e)$. 